\title{
Tratamiento de Infecciones Urinarias en Niñas con Trimetoprim-Sulfametopirazina.
}

\author{
Dr. Waldo Rodriguez Y.1, Dr. Carlos Delucchi Z. 2 , Dr. M. Antonio Bidegain S. ${ }^{3}$ \\ Treatment of Tract Infections in Children with \\ Trimethoprim - Sulfamethopirazine
} Dra. M. Soledad Rodríguez T. ${ }^{1}$ - B, Dra. Andrea Gleisner E. ${ }^{1}$ - B, Dra. Sonia Fígueroa Y. ${ }^{1}$

\begin{abstract}
20 female patients, aged 1 to 14 years (average 7.8 years), who underwent acute or recurrent urinary tract infections have been treated with. Trimethoprim-Sulfamethopirazine (TMP-SMPz) (pediatric syrup, $1.8 \%$ for ten days, $0.4 \mathrm{ml} / \mathrm{kg}$. every 12 hours. Before treatment urine cultures were realized in order to identify bacteria and sensibility to TMP - SMPz, in order to compare it with Cotrimoxazole. E. coli resulted to be the etiologic agent in 19 cases and $P$. mirabilis in one. The sensibility to the drug was optimal in all patients and not significantly different to cotrimoxazole. Clinjcal results showed great improvement in acute and recurrent urinary tract infections in $24-$ $4 B$ hours, and negative cultures at the 5 th day of treatment. Cultures repeated 10 and 17 days after start of treatment were negative. The drug was well tolerated except fot one patient with gastrointestinal symptoms, one with eosinophylia and a third one with low white blood cells counts and elevation of serum Gutamic-Piruvic transaminase that recovered spontaneously.
\end{abstract}

Las infecciones urinarias siguen constituyendo una patología importante en Pediatría, no sólo por su frecuencia, sino por el riesgo potencial que irivolucran. El diagnóstico oportuno, el correcto manejo y el adecuado seguimiento de ellas permitiría reducir la incidencia de daños renales posteriores ${ }^{1-2-3}$.

El correcto manejo de las infecciones del tracto urinario reside en el uso oportuno y adecuado de diversas drogas antimicrobianas, entre las que destaca desde hace algunos años la asociaciốn quimioterápica de trimetoprim (TMP) con una sulfa, el sulfametoxazol (SMX) denominada Cotrimoxazol (CTX), en una relación de $1: 5$. Esta asociación constituye hoy una de las elecciones para el tratamiento oral en este tipo de infecciones con gérmenes sensibles a ella ${ }^{4-5-6}$

En la búsqueda de una mayor efectividad y tolerancia de esta forma de asociación antimicrobiana, se ha sintetizado un nuevo compuesto constituido por el trimetoprin y otro tipo de sulfa, la sulfametopirazina (SMPZ), de acción más prolongada que las sulfas anteriores, en una relación de 5:4 (TMP-SMPZ) ${ }^{7}$.

La nueva asociación quimioterápica ha sido utilizada en diversas experiencias clinicas, tanto nacionales como extranjeras, demostrando una elevada efectividad terapéutica en procesos tales como infecciones urinarias, respiratorias, gastrointestinales, etc. ${ }^{8-9}$.

1 Departamento de Pediatría y Medicina.

2. Facultad de Medicina de la Universidad de Concepción.

3 Servicio de Pediatría Hospital Guillermo Grant Benavente - Concepción.

l-B Residente Becario.
Debido a la reciente disponibilidad de este fármaco en forma de jarabe para uso pediátrico al $1,8 \%$ hemos querido evaluar en esta investigación el comportamiento microbiológico de los gérmenes obtenidos de niños portadores de infecciones urinarias agudas, recurrentes o ambas, comparando la sensibilidad del TMP-SMPz con el cotrìmoxazol, y la evolución clínica y bacteriológica de estas infecciones urinarias tratando a los pacientes con TMP - SMPz, analizando la eficacia terapéutica, tolerancia y efectos colaterales de la droga.

\section{PACIENTES Y METODOS}

Se estudiaron 20 niñas mayores de 1 año $y$ menores de 14 años de edad (edad medja 7,8 años), 10 de las cuales sufrían infecciones urinarias y otras 10 recurrencias. Todas provenian de la Unidad de Nefrourologia del Servicio de Pediatria del Hospital Guillermo Grant Benavente y de las clinicas privadas de Concepción y consultaron en un periodo de ocho meses.

Antes de iniciar el tratamiento en cada pacien. te se identificó el germen causal y su sensibilidad TMP-SMPz y Cotrimoxazol de la siguiente forma:

Las muestras fueron tomadas bajo rigurosas normas de asepsia y procesadas en el laboratorio antes de transcurridos 30 minutos de su obtención. Se sembraron en caldo de Müller-Hinton y su sensibilidad se determinó mediante la prueba de Bauer-Kirby, utilizando discos con $3 \mathrm{mcg}$. de TMP y $30 \mathrm{mcg}$. de SMPz. Los discos de Cotrimoxazol contenían $1,25 \mathrm{mcg}$. de TMP y 23,75 mcg. de SMX. 
Se consideró que el germen exa muy sensible cuando se encontró un halo de inhibición de 15 mm., medianamente sensible de 11 a $15 \mathrm{~mm}$. y resistente en los casos de menos de $11 \mathrm{~mm}$.

A los 5,10 y 17 días después de iniciado el tratamiento se realizaron estudios bacteriológicos de control.

En todos los pacientes los gérmenes resultaron sensibles al TMP-SMPz y se evaluó la eficacia terapéutica del fármaco, que fue administrado en forma de jarabe, en dosis de $0,4 \mathrm{ml} \mathrm{kg}$., cada 12 horas durante 10 días. Esta forma farmacéutica contiene por cada ml., $10 \mathrm{mg}$. de trimetoprim y $8 \mathrm{mg}$. de sulfametopirazina, es decir, $1 \%$ de trimetoprim y $0,8 \%$ de sulfametopirazina, respectivamente. La dosificación pediátrica recomendada es de 8 y $6,4 \mathrm{mg} / \mathrm{kg} / \mathrm{d}$ ja de trimetoprim $y$ sulfametopirazina, respectivamente, fraccionado cada 12 horas.

En todos los casos se practicó hemograma, proteinemia; uremia; creatininemia; velocidad de sedimentación globular; deshidrogenasa láctica y transaminasas GOT, GPT séricas antes y al $10^{\circ}$ dia de tratamiento. Los perfiles urinarios como en el caso de los estudios microbiológicos se realizaron al ingreso, al $5^{\circ}, 10^{\circ}$ y $17^{\circ}$ dia de evolucion, en el Laboratorio de Exploración Renal del Centro Nefrourológico de Concepción.

Todas las pacientes que presentaron infecciones urinarias recurrentes, fueron sometidas a urografía excretora y uretrocistografía retrógrada. Los criterios que se consideraron para evaluar la actividad del fármaco fueron la evolución de los sintomas de los urocultivos al $5^{\circ}, 10^{\circ}$ y $17^{\circ}$ dia de iniciada la terapia.

\section{RESULTADOS}

Estudio de Sensibilidad:

Los gérmenes aislados fueron Escherichia coli en 19 ocasiones y Proteus mirabilis en una.

La sensibilidad de estas cepas al TMP-SMPz y Cotrimoxazol se detalla en la Tabla 1.

Del análisis de estos resultados se desprende que no hay diferencias significativas entre los halos inhibitorios del desarrollo microbiano producidos por ambos medicamentos.

Los rangos obtenidos fueron de $27-36 \mathrm{~mm}$. para TMP-SMPz y $26-33 \mathrm{~mm}$. para el Cotrimoxazol.

En todos los casos las bacterias aisladas han resultado muy sensibles a ambos fármacos.

Eficacia Clínica y Tolerabilidad:

Formas Agudas: Comprendió 10 enfermas con una edad promedio de 6 años 10 meses y síntomas urinarios en todas, consistentes en compromiso del estado general, fiebre, polaquiuria y disuria.

Los controles clínicos realizados a las 48 horas

Tabla 1.

Sensibilidad Bacteriana a Trimetoprim-Sulfametopirazina y Cotrimoxazol expreada en mm. de halo inhibitorio*

$\begin{array}{ccccc}\text { Paciente } & \text { Germen } & \text { Recuento } & \text { TMP-SMPz } & \text { Cotrimoxazol } \\ 01 & & & & \\ 02 & \text { E. coli } & 10^{7} & 31 \mathrm{~mm} . & 31 \mathrm{~mm} . \\ 03 & \text { E. coli } & 10^{7} & 31 \mathrm{~mm} . & 30 \mathrm{~mm} . \\ 04 & \text { E. coli } & 10^{7} & 28 \mathrm{~mm} . & 28 \mathrm{~mm} . \\ 05 & \text { E. coli } & 10^{7} & 30 \mathrm{~mm} . & 29 \mathrm{~mm} . \\ 06 & \text { E. coli } & 10^{7} & 29 \mathrm{~mm} . & 28 \mathrm{~mm} . \\ 07 & \text { E. coli } & 10^{7} & 34 \mathrm{~mm} . & 32 \mathrm{~mm} . \\ 08 & \text { E. coli } & 10^{7} & 31 \mathrm{~mm} . & 29 \mathrm{~mm} . \\ 09 & \text { E. coli } & 10^{7} & 32 \mathrm{~mm} . & 31 \mathrm{~mm} . \\ 10 & \text { E. coli } & 10^{7} & 32 \mathrm{~mm} . & 27 \mathrm{~mm} \text {. } \\ 11 & \text { E. coli } & 10^{7} & 28 \mathrm{~mm} . & 29 \mathrm{~mm} . \\ 12 & \text { E. coli } & 10^{7} & 32 \mathrm{~mm} . & 32 \mathrm{~mm} . \\ 13 & \text { E. coli } & 10^{6} & 30 \mathrm{~mm} . & 32 \mathrm{~mm} . \\ 14 & \text { E. coli } & 10^{7} & 36 \mathrm{~mm} . & 28 \mathrm{~mm} . \\ 15 & \text { P. mirabilis } & 10^{7} & 32 \mathrm{~mm} . & 33 \mathrm{~mm} . \\ 16 & \text { E. coli } & 10^{7} & 30 \mathrm{~mm} . & 33 \mathrm{~mm} . \\ 17 & \text { E. coli } & 10^{7} & 34 \mathrm{~mm} . & 29 \mathrm{~mm} . \\ 18 & \text { E. coli } & 10^{7} & 34 \mathrm{~mm} . & 31 \mathrm{~mm} . \\ 19 & \text { E. coli } & 10^{7} & 30 \mathrm{~mm} . & 26 \mathrm{~mm} .\end{array}$

$*$ MUY SENSLBLE $=$ Halo $15 \mathrm{~mm}$. 
de iniciado el tratamiento, demostraron ausencia de síntomas en todas las enfermas.

Los cultivos urinarios practicados al $5^{\circ}, 10^{\circ} \mathrm{y}$ $17^{\circ}$ día de evolución resultaron todos estériles.

La tolerancia a! medicamento fue buena, salvo en una niña de 3 años de eđad que presentó intoletancia gástrica al TMP-SMPz por lo que hubo que suspenderlo al $4^{\circ}$ día de iniciado el tratamiento. En el momento de suspender el fármaco, la paciente estaba asintomática y los controles bacteriológicos de orina fueron negativos al $5^{\circ}, 10^{\circ}$ y $17^{\circ}$ día.

No se observaron cambios de importancia en los exámenes de laboratorio practicados al ingre. so $y$ al término del tratamiento.

Formas Recurrentes: Se evaluaron 10 pacientes, con una edad promedio de 8 años 5 meses.

En este grupo hubo 4 ninlas que presentaron compromiso del estado general, fiebre, polaquiuria y disuria. Orina de mal olor en dos casos, uno de los cuales turvo fiebre. Dos niñas sólo presentaron polaquiuria y dos pacientes estaban clínicamente asintomáticas y se comprobó la recurrencia urinaria sólo por los resultados de los exámenes de laboratorio, en forma reiterada.

La exploración radiológica en este grupo de pacientes, demostró que el $40 \%$ de las urografías excretoras y el $50 \%$ de las uretrocistografías retrógradas, mostraban alteraciones anatómicas que explicaban las recidivas. Las más frecuentes fueron hidronefrosis (dos casos), hipoplasia renal (un caso), doble sistema pielocaliciario (un caso), vejiga neurogénica (dos casos), reflujo vesicoureteral. (un caso) y alteraciones ureterales (dos casus).

La evolución clínica de este grupo de pacientes demostró que a las 24 horas de iniciada la terapia con TMP $-\mathrm{SMPz}$, nueve enfermas estaban asintomáticas y solamente una persistió con orinas de mal olor que desapareció al $2^{\circ}$ día de tratamiento.

En los controles bacteriológicos realizados durante el tratamiento con TMP-SMP2 se pudo comprobar que todas las pacientes tuvieron exá. menes urinarjos normales y cultivos estériles al $5^{\circ}$ día de tratamiento los cuales se mantuvieron en los controles posteriores.

La tolerancia al medicamento fue excelente en los 10 casos, destacando solamente algunas modjficaciones en los exámenes de laboratorio en dos pacientes. La primera tuvo al término de tratamiento una eosinofilia moderada cuya relación con el fármaco es posible, aunque no se puede descartar otras causas como alergias, parasitosis, etc. La segunda enferma, experimentó al término de la investigación, un recuento leucocitario, límite con 3.800 glóbulos blancos por $\mathrm{mm}^{3}$. y una elevación transitoria de las S.G.P.T de 25 U/1 a $71 \mathrm{U} / 1$ las cuales se normalizaron en los contro. les posteriores efectuados al mes de finalizada la experiencia.

\section{COMENTARIO}

Nuestro primer propósito de investigación fue estudiar comparativamente el cormportamiento de TMP-SMPz del Cotrimoxazol, mediante la medición del halo inhibitorio frente a cepas bacterianas procedentes de pacientes portadores de infecciones urinarias. Los resultados demostra. ron que la sensibilidad de los agentes bacterianos a estos fármacos es prácticamente similar. Los halos inhibitorios en las placas de cultivo no mostraron diferencias significativas entre ambas drogas.

En nuestro material de estudio pudimos comprobar la eficacia terapéutica que muestra TMP-SMPz en el manejo de las infecciones urinarias, agudas y recurrentes, cuando los gérmenes encontrados son sensibles al fármaco. Así, se pudo demostrar que las pacientes mejoraron clínicamente en todos estos casos después de 24 a 48 horas de iniciar la administración del fárma. co. Similar eficacia se pudo observar en los controles de los perfiles urinarios y urocultivos realizados al $5^{\circ}, 10^{\circ}$ y $17^{\circ}$ día de iniciada la investigación.

Estos resultados, permiten plantear como una alternativa interesante, tratamientos con este tipo de fármacos por períodos más cortos, siempre que se pesquisen gerrmenes sensibles y no exista evidencia de darto renal, como puede ocurrir en infecciones crónicas del tracto urinario 10-11-12.

Por otra parte, es preciso destacar la eficacia del medicamento en pacientes con infecciones recurrentes, donde se comprobó malformaciones del árbol urinario. Las características de estos enfermos, en que la resistencia bacteriana suele ser alta, confirman la favorable actividad del quimioterápico en estudio, cuando el germen pesquisado es sensible.

La tolerancia de la droga fue buena en el $95 \%$ de los casos:

En esta experiencia no se presentaron matifestaciones clinicas de reacciones cutáneas.

En los exámenes de laboratorio se pesquisaron algunos efectos colsterales, como eosinofilia moderada en una paciente $y$ en otro caso una alteración de mayor severidad consistente en leucopenia y elevaciones de las transaminasas, $1 a$ que regresaron espontáneamente a lo normal un mes después.

Nuestros resultados en procesos agudos y recurrentes, confirman en el nifio, la eficacia $y$ 
buena tolerancia ya documentadas en las infecciones de las vías urinarias del adulto $13 \cdot 14$.

En dos trabajos recientes, se ha comunicado el tratamiento de un limitado número de pacientes en edad pediátrica con TMP-SMPz jarabe.

Weippl y cols. ${ }^{15}$ en una experiencia clínica comparativa entre $\mathrm{TMP}-\mathrm{SMPz}$ y Cotrimoxazol ha obtenido en 12 pacientes con infecciones agudas o recurrentes del tracto urinario la cura. ción clínica y esterilización de los urocultivos en todos los sujetos tratados. Como en los casos ya descritos, el fármaco ha demostrado una elevada eficacia (esterilización de todos los cultivos al $4^{\circ}$ día) superior a aquella del Cotrimoxazol utilizado por estos autores como fármaco de control.

G. Weippl y cols. ${ }^{16}$ en un estudio multicéntrico de 91 casos, ha tratado a 9 pacientes con infecciones agudas o recurrentes del aparato urjnario con TMP-SMPz jarabe pediátrico, obteniendo la curación clínica y bacteriológica en 8 .

El esquema posológico empleado en ambos casos ha sido semejante al utilizado por nosotros, vale decir $0,4 \mathrm{ml}$ por $\mathrm{kg}$. cada 12 horas.

El uso de dos dosis diarias del fármaco en el niño a diferencia de una sola (después de una dosis doble de ataque el primer dia) en el adulto, se justifica debido a los hallazgos encontrados en la farmacocinética del $\mathbf{T M P}-\mathrm{SMPz}$ en ambas edades. De hecho, en el niño tanto el Trimetoprim como la Sulfametopirazina se eliminan más rápidamente (media plasmática de 6-40 horas repectivamente) que en el adulto (12 y 60 horas), por tanto, parece necesario dar dos dosis para obtener niveles terapéuticamente eficaces en la sangre y en la orina ${ }^{17}$.

Como conclusión podemos afirmat que nuestros resultados confirman la limitada experiencia presentada en otras publicaciones en donde el TMP-SMPz en las dosis empleadas por nosotros es un fármaco eficaz y bien tolerado por el niño, que puede ser utilizado tanto en las infecciones agudas como en las recurrentes del aparato urinario.

\section{RESUMEN}

20 niñas de edades entre 1 y 14 años (promedio 7,8 años), portadoras de infecciones agudas - recurrentes del tracto urinario fueron tratadas con una combinación de Trimetoprim-Sulfametopirazina (jarabe pediátrico al $1,8 \%$ ), por 10 dias a la dosis de $0,4 \mathrm{ml} / \mathrm{kg}$. cada 12 horas.

Antes de iniciar el tratamiento se realizaron urocultivos con el fin de identificar los agentes patógenos y verificar la sensibilidad del TMP-SMPz, en comparación con Cotrimoxazol.

En 19 casos, E. coli resultó ser el agente etiológico y en un caso el P. mirabilis. En todos los pacientes la sensibilidad de los gérmenes al fármaco fue buena y no signifjcativamente diferente a la del Cotrimoxazol.

Los resultados clínicos indicaton que en todos los casos tratados tanto en las formas agudas como en las recurrentes ha habido una rápida mejoría de los síntomas entre las 24 y las 48 horas y esterilización de Jos cultivos antes del $5^{\circ}$ día de tratamiento.

Los controles bacteriológicos realizados al $10^{\circ}$ y $17^{\circ}$ día de inicio del tratamiento fueron negativos. La tolerancia de las niñas a las drogas fue buena en general, excepto en una paciente que tuvo molestias digestivas, un caso en que hubo eosinofilia y otro que presentó leucopenia y elevación de las transaminasas del suero que se recuperaron espontáneamente.

\section{AGRADECIMIENTOS}

Los autores agradecen a Farmitalia Carlo Erba Laboratorio S.A. que proporcionó el medicamento (KELFIPRIM ${ }^{\circledR}$ que hizo posible esta investigación.

\section{REFERENCIAS}

1 Edeiman Chester: Pediatric Kidney Disease. Little, Brown and Company Boston. Vol. Il, 1978.

2 Hollerman Charles: Pediatric Nephrology. Medical Examination Publishing Co., Inc. New York, 1979.

3 Lagomarsino Edde: Nefrología. Editorial Andrés Bello, Santiago, 1982

4 Goodman and Gilman: The Pharnacological Basis of Therapeutics. Macmillan Publishing C., Inc., 1980.

${ }^{5}$ Kasonen A., Sundquist $H$. and Jummila S.Y.T.: Trimethoprim in the treatment of acute urinary tract infection. Excerpta Medica. Urology and Nephrology 14: 182 Abstract 1072, 1980.

${ }^{6}$ Ortengren B., Magni L, and Bertan $T$.: Development of sulphonanide-trimethoprim combinations for urinary tract infections.

Pant 3: Pharmacokinetic characterization of sulphadiazine and sulphamethoxazole given with trimethoprim. Excerpta Medica. Urology and Nephrology 15: 429 Abstracts 2532, 1980.

${ }^{7}$ Minocci D., Martinengo $C$., Pretti $G$.: Sensibilita in vitro alla associazione trime thoprim-sulfametopirazina di batteri isolati da urina. Atti della Accad., Med. Lombardi XXX1V, 1979.

Prado V., Aguire X., Pinto M.E. y Giglic M.S.: Sensibilidad in vitro (CIM) en bacilos Gtam (-) frente a la asociación Trimetoprim-Sulfametopirazina (Kelfiprim). Rev. Med. de Chile 110: 29, 1982.

${ }^{9}$ Cortez $M$. . Vallejos $T$. . Vargas $F$. , Csendes 1. : Trumetoprim más Sulfametopirazina (Kelfiprim) en infección urinaria. Rev. Med. de Chile 110: 453, 1982.

10 Khan A., Kumar K. and Evons H.J.: Three-day antimicrobial therapy of urinary ttact infections. J. Pediat. 99: 992, 1981.

11 Lohr J, Joyden G., Kessler $R$, et al.: Three-day therapy of lower urinary tract infection with nitrofusantoin marcrecristal: A tandomized clinical trial. J. Pediat. 99: 980, 1981. 
12 Bergors G.: Clinical experience of Kelfizina in urinary tract infections in children. Comunicated at the Round Table on Kelfizina. Viena, Junio 19, 1967 (5th Internat. Congress of Chemotherapy).

13 Grazioni $G$. et al.: Kelfiprim vs Cotrimoxazole in recurrent and persistent urinary tract infections. Multicentre double blind trial. Presentado al 40 Congreso di Urología, Atene 27-31 Maggio, 1980.

19 Pisani $E$. et al: Kelfiprim, a new sulpha-trimethoprim combination versus Cotrimoxazole, in the treatment of urinary tract infections; a multicentre, double blind trial. Urological Research, 10, 41-44, 1982.

15 Weippl G., Marrl E., Rosenfranz A. ef al.: Treatment with Kelfiprim sycup of various infectious conditions in infants. A multi-institutional study on 91 cases. (comunicación personal)

16 Weippl G., Petrescu D., Grippi W. et al, Eficacia c Tolerabilidade de Kelfiprim suspensao no Tratamento de Processos Infeccciosos em Criancas. Resultados preliminares de 72 casos, num Estudo Comparativo vs. Cotrimoxazol, Ampicilina e Eritcomicina. Rev. Bras, Med. 38 (9), 1981.

17 Bassetti D. et al.: Phamacokinetic study of a new sulphamethopyrazine trimethoprim combination in children. Current Chemotherapy and Immunotherapy. Proceedings of 12 th International Congress of Chemotherapy, Florence 19--24 Jily/1981, Vol. I pp. 242-243. 